\title{
System Consolidation of Spatial Memories in Mice: Effects of Enriched Environment
}

\author{
Joyce Bonaccorsi, ${ }^{1,2}$ Simona Cintoli, ${ }^{2,3}$ Rosa Mastrogiacomo, ${ }^{3}$ Sigrid Baldanzi, ${ }^{3}$ \\ Chiara Braschi, ${ }^{2}$ Tommaso Pizzorusso, ${ }^{2,4}$ Maria Cristina Cenni, ${ }^{2}$ and Nicoletta Berardi ${ }^{2,4}$ \\ ${ }^{1}$ Scuola Normale Superiore, Piazza dei Cavalieri 7, 56100 Pisa, Italy \\ ${ }^{2}$ Institute of Neuroscience of the CNR, 56124 Pisa, Italy \\ ${ }^{3}$ Pisa University, 56100 Pisa, Italy \\ ${ }^{4}$ Department of Neuroscience, Psychology, Drug Research and Child Health, NEUROFARBA, Università di Firenze, \\ Viale Pieraccini, 50100 Firenze, Italy
}

Correspondence should be addressed to Nicoletta Berardi; berardi@in.cnr.it

Received 4 April 2013; Accepted 15 June 2013

Academic Editor: Andrea Guzzetta

Copyright (C) 2013 Joyce Bonaccorsi et al. This is an open access article distributed under the Creative Commons Attribution License, which permits unrestricted use, distribution, and reproduction in any medium, provided the original work is properly cited.

\begin{abstract}
Environmental enrichment (EE) is known to enhance learning and memory. Declarative memories are thought to undergo a first rapid and local consolidation process, followed by a prolonged process of system consolidation, which consist in a time-dependent gradual reorganization of brain regions supporting remote memory storage and crucial for the formation of enduring memories. At present, it is not known whether EE can affect the process of declarative memory system consolidation. We characterized the time course of hippocampal and cortical activation following recall of progressively more remote spatial memories. Wild-type mice either exposed to EE for 40 days or left in standard environment were subjected to spatial learning in the Morris water maze and to the probe test $1,10,20,30$, and 50 days after learning. Following the probe test, regional expression of the inducible immediate early gene c-Fos was mapped by immunohistochemistry, as an indicator of neuronal activity. We found that activation of the medial prefrontal cortex (mPFC), suggested to have a privileged role in processing remote spatial memories, was evident at shorter time intervals after learning in EE mice; in addition, EE induced the progressive activation of a distributed cortical network not activated in non-EE mice. This suggests that EE not only accelerates the process of $\mathrm{mPFC}$ recruitment but also recruits additional cortical areas into the network supporting remote spatial memories.
\end{abstract}

\section{Introduction}

Environmental enrichment (EE) is an experimental protocol classically defined as "a combination of complex inanimate and social stimulation" [1] and which provides animals with the opportunity to attain high levels of voluntary physical activity on running wheels and to enhance exploration, cognitive activity, and social interaction. Several studies point out that animals reared in EE show marked brain changes at functional, anatomical, and molecular levels [2-13] and in particular changes in plasticity factors and mechanisms $[14,15]$. EE can indeed be used as a noninvasive strategy to modulate brain plasticity throughout life; EE can accelerate the development of the central nervous system [16-18], can reopen plasticity windows in the adult cortex $[19,20]$ and causes a significant improvement in learning and memory $[14,21-23]$, especially evident in aged animals [22, 24-32], or in animal models of neurodegenerative diseases [33-35].

Declarative memories depend initially on the medial temporal lobe system, including the hippocampus, but, over days to weeks, as these memories mature, they become increasingly dependent on other brain regions such as the neocortex [36-39]. This process of time-dependent gradual reorganization of the brain regions that supports remote memory storage and underlies the formation of enduring memories, is known as system-level memory consolidation or system consolidation [40,41]. It has been demonstrated that the progressive stabilization of long-lasting memories is 
due to the reactivation of hippocampal-cortical connections $[42,43]$ and the strengthening of corticocortical connections, involving cortical plasticity mechanisms [41, 44-48]. Despite all the evidences showing that EE enhances cortical plasticity and learning and memory, there is no evidence on whether and how EE could affect the process of time-dependent system consolidation.

This study aimed at testing whether EE could affect the system-level memory consolidation process using a spatial memory paradigm and characterizing the time course of hippocampal and of cortical activation following recall of progressively more remote memories.

Spatial memory is a declarative type of memory. The hippocampus plays an essential role in the formation of spatial memories [49-53]; subsequently, spatial memories become, in a gradual manner, additionally dependent on other cortical regions. Many studies point out that the privileged final storage site for remote spatial memories is the prefrontal cortex (PFC) and in particular the medial PFC (mPFC), including the anterior cingulate, prelimbic, and infralimbic cortices $[47,48,54,55]$. Recently, however, it has been suggested that remote memories recall involves the coordinated activation of a broader network of cortical brain regions [56-58]. We have therefore characterized the time course of activation of a number of cortical areas in addition to the MPFC. We found that EE not only induces an earlier recruitment of mPFC, but also induces the progressive activation of a distributed cortical network that is not activated in standard housed mice.

\section{Materials and Methods}

2.1. Animal Treatment. Male and female C57BL/6 mice of 2 months of age were used in this study. All the procedures were approved by the Italian Ministry of Health. The animals were housed in an animal room with a $12 \mathrm{~h} / 12 \mathrm{~h}$ light/dark cycle, with food and water available ad libitum. At 2 months of age, the animals were assigned to one of the following rearing conditions for 40 days: environmental enrichment (EE, $n=24$ ) or standard condition (SC, $n=24$ ). The SC rearing condition consisted of a $26 \times 18 \times 18 \mathrm{~cm}$ cage housing 3 animals. The EE rearing condition was achieved using a large cage $(44 \times 62 \times 28 \mathrm{~cm})$ containing several food hoppers, one running wheel for voluntary physical exercise, and differently shaped objects (tunnels, toys, shelters, stairs) that were repositioned twice a week and completely substituted with others once a week [33]. Two additional groups of control animals, age and gender matched to $\mathrm{SC}$ and $\mathrm{EE}$ groups, were housed in home cage standard condition (HC-SC, $n=8$ ) or in home cage enriched condition (HC-EE, $n=7$ ), and they did not perform any behavioural task.

2.2. Morris Water Maze (MWM). The hidden platform version of the MWM test was performed [59]. A large water tank of $120 \mathrm{~cm}$ of diameter was filled with white opaque water at $22^{\circ} \mathrm{C}$. An escape platform of $11 \mathrm{~cm}$ of side was submerged $1 \mathrm{~cm}$ below the water surface and placed in the center of the SW quadrant. The platform was maintained in this position for all the swim trials through the test. Mice were trained to swim to the platform in 4 daily trials, starting in pseudorandomly varied locations, with a $30 \mathrm{~min}$ interval, during 7 consecutive days. The trial was complete once the mouse found the platform or $60 \mathrm{sec}$ had elapsed. If the mouse failed to find the platform on a given trial, the experimenter guided the mouse onto the platform. Once reaching the platform, each mouse was allowed to rest for $20 \mathrm{~s}$ on it. After each trial each mouse was returned to its home cage where it rested until the next trial. After the completion of training, spatial memory was assessed in a probe test; a recall probe trial was performed after $1,10,20,30$, and 50 days after the end of learning. We used an automated tracking system (Noldus Ethovision XT) for recording behavioural data from training and probe tests. For each trial we measured the latency (in sec) to reach the platform, the total distance (in $\mathrm{cm}$ ) swam in order to reach the platform, and the average swim speed (in $\mathrm{cm} / \mathrm{s}$ ). For each probe trial we measured the amount of time spent in the target zone $(23 \mathrm{~cm}$ in radius, centered on the location of the platform during training) and the average time spent in three other equivalent zones in each quadrant $[55,60]$.

2.3. Immunohistochemistry. Mice were anaesthetized and perfused via intracardiac infusion with $0.1 \mathrm{M}$ PBS and then $4 \%$ paraformaldehyde (PFA, dissolved in $0.1 \mathrm{M}$ phosphate buffer, $\mathrm{pH}$ 7.4) $90 \mathrm{~min}$ after the completion of behavioral testing. Brains were removed, fixed overnight in PFA, and then transferred to $30 \%$ sucrose solution and stored at $4{ }^{\circ} \mathrm{C}$. Coronal sections were cut at $40 \mu \mathrm{m}$ thickness on a freezing microtome (Sliding Leica microtome SM2010R, Leica Microsystems), and free-floating sections were prepared for immunohistochemistry. After a blocking step in 10\% NGS and $0.5 \%$ Triton X-100 in PBS, sections were incubated in a solution containing $1 \%$ NGS, $0.3 \%$ Triton X-100, and anti-cFos primary rabbit polyclonal antibody $(1: 3000$ rabbit anti c-Fos polyclonal antibody, Calbiochem, USA) for $36 \mathrm{~h}$ at $4^{\circ} \mathrm{C}$. Subsequently, sections were transferred in a solution containing 1\% NGS, $0.1 \%$ Triton X-100, and 1:200 anti-rabbit biotinylated secondary antibody (Vector Labs) in PBS. This was followed by incubation in ABC kit (Vector Labs) and final detection with $\mathrm{DAB}$ reaction kit (Vector Labs). Sections were finally mounted on gelatinized slides, dehydrated, and sealed with DPX mounting medium (VWR International, UK).

2.4. Analysis of c-Fos Positive Cells. Counting of c-Fos positive cells in different brain areas was performed using a CCD camera (MBF Bioscience, Germany) mounted on a Zeiss Axioskop (Zeiss, Germany) microscope and the StereoInvestigator software (MBF Bioscience). Brain structures were anatomically defined according to a mouse brain atlas (Paxinos and Franklin [61]), and the regions of interest selected for measurement of c-Fos-positive nuclei were (numbers indicate the distance in millimeters of the sections from bregma) infralimbic cortex (IL, $+1.94 \mathrm{~mm}$ ); secondary motor cortex $(\mathrm{M} 2,+0.98 \mathrm{~mm})$; anterior cingulate cortex, area 1 and area $2(\mathrm{aCC},+0.98 \mathrm{~mm}$ ); dentate gyrus ( $\mathrm{DG},-1.94 \mathrm{~mm})$; CA1 field of dorsal hippocampus (dCA1, $-1.94 \mathrm{~mm}$ ); CA3 field of dorsal hippocampus (dCA3, $-1.94 \mathrm{~mm}$ ); posterior 
parietal association cortex (pPtA, $-1.94 \mathrm{~mm})$; primary auditory cortex (Aul, $-3.16 \mathrm{~mm}$ ); primary visual cortex (V1, $-4.16 \mathrm{~mm}$ ); medial entorhinal cortex (MEnt, $-4.16 \mathrm{~mm}$ ). The number of c-Fos-positive cells was counted at 20x magnification, following a "blind procedure", in 10-40 fields $(50 \times$ $50 \mu \mathrm{m}$ or $100 \times 100 \mu \mathrm{m})$ per section according to the size of brain structure and their density calculated (cells $/ \mathrm{mm}^{2}$ ), using at least 5 sections for each structure.

2.5. Statistics. All results were expressed as mean \pm s.e.m., and all statistical analysis were performed using statistical software package SigmaStat. For MWM performance in the learning phase, a two-way analysis of variance (ANOVA) for repeated measures (RM) was performed, considering both factor housing condition (EE or SC) and factor learning day, with post hoc analysis Holm-Sidak method. Performance in each probe test was compared with one-way ANOVA across circular zones (target zone versus the average of other zones) for each housing condition. The c-Fos protein expression in each area was analyzed with a two-way ANOVA for housing condition factor and probe test day factor, with post hoc analysis Holm-Sidak method.

\section{Results}

3.1. Hippocampus Is Activated following Spatial Memory Recall at All Temporal Points Tested Both in EE and SC Mice. To test whether EE can affect the system consolidation process, we trained C57BL/6 mice, housed in different conditions (standard condition, SC $n=24$ or environmental enrichment $\mathrm{EE} n=24$ ), in a spatial learning task, using the Morris water maze, and we analyzed the following parameters referred to the average of 4 daily trials, during 7 consecutive days: latency to find the platform $(\mathrm{s})$, total distance swam $(\mathrm{cm})$, and mean swim speed $(\mathrm{cm} / \mathrm{s})$. For the distance swam and the latency to reach the platform during acquisition, a significant learning effect for both housing conditions was found, but not a significant difference between the two groups (two-way RM ANOVA, for latency $P=0.016$; for distance swan $P<0.001$ ). For the latency parameter only, we found a housing condition $x$ day interaction: multiple comparisons showed that the main differences resided on days 4 and 5 (Figure 1(a)) (Twoway RM ANOVA, post hoc analysis Holm-Sidak method, for day $4 P=0.005$; for day $5 P=0.001$ ). We also measured the mean swim velocity throughout the test, in order to exclude differences in navigation speed (data not shown): we observed a significant decrease in the mean swim velocity through the test (two-way RM ANOVA, $P<0.001$ ), but neither a difference between housing condition $(P=0.276)$ nor a housing condition $\times$ day interaction $(P=0.163)$.

Spatial memory was evaluated in a probe test in which the hidden platform was removed. We performed recall probe tests at 1, 10,20,30, and 50 days, and we quantified exploration in the target zone, a circular zone (radius: $23 \mathrm{~cm}$ ) in quadrant where the platform was placed during training, and the average time spent in three other equivalent zones in each quadrant, for SC and EE mice (Figure 1(b)) [55, 60]. We found a significant difference between target zone versus the others in probe tests at $1,10,20$, and 30 days, for both groups (see Figure 1(b) for details).

After the probe test, mice were sacrificed and the protein c-Fos was immunolabeled as an indicator of neuronal activity. c-Fos expression was calculated as the density of number of c-Fos-positive cells in $\mathrm{mm}^{2}$. First we investigated cFos expression in the hippocampus, the structure that is responsible for the formation of long term spatial memory [49-53].

Levels of c-Fos expression in the hippocampus of control mice (Home cage mice, HC-EE and HC-SC mice) did not differ between housing conditions (HC-SC $n=8$; HC-EE $n=7$; one-way ANOVA, $P=0.736$ ); levels of c-Fos protein for EE and SC mice were significantly greater than those in their home-cage controls at all retention intervals, (two-way ANOVA, post hoc analysis Holm-Sidak method, all $P$ values $<0.05$ ), suggesting that the hippocampus is involved both in the formation and delayed recall of the spatial memory. We found a similar c-Fos expression in the EE and SC mice in all probe tests (two-way ANOVA, post hoc analysis Holm-Sidak method for housing condition, $P=0.731$ ).

We then focused on the dorsal hippocampus (dHCP), known to be specifically involved in spatial memory [60]; again, we observed the same c-Fos expression pattern in EE and SC group; activation increased with increasing retention interval up to 30 days (see Figure 2 for details).

3.2. EE Induces an Early Recruitment of the MPFC. The results for c-Fos expression in the mPFC, the final memory storage site in the cortex, show that both the aCC and the IL have the same time course of c-Fos protein expression pattern; cfos expression at 1 day did not differ from that in home cage animals, both for $\mathrm{EE}$ and $\mathrm{SC}$ mice, and there was no difference between EE and SC or HC-EE and HC-SC mice (twoway ANOVA, post hoc analysis Holm-Sidak method, all $P$ values $>0.05$ ); however, starting from the probe test at 10 days, c-Fos expression was greater in EE group than HC-EE and SC groups, with a further increase at 20 days (see Figure 3 for details); only for $\mathrm{EE}$ animals did the c-Fos protein expression differ from that of HC control animals. For the probe tests at 30 and 50 days we found that the c-Fos expression in SC animals differed from that of HC-SC animals; values of SC and $\mathrm{EE}$ groups did not differ (two-way ANOVA, post hoc analysis Holm-Sidak method; see Figure 3 for details).

\subsection{EE Induces the Involvement of Distributed Cortical Net-} work in Supporting Remote Spatial Memory. To examine the time-dependent reorganization of neuronal activation in a brain-wide manner, we observed c-Fos protein expression in other cortical areas that are important for the construction of spatial maps. Using several techniques, Wang et al. [62] determined that distinct areas of extrastriate visual cortex are gateways for ventral and dorsal streams in the mouse. The dorsal stream includes the network hippocampus-medial entorhinal cortex [63]-posterior parietal cortex [64] for spatial navigation; in addition, the dorsal stream is connected to auditory cortex and to frontal areas, such as cingulate cortex, infralimbic cortex, and motor areas [62]. First we 


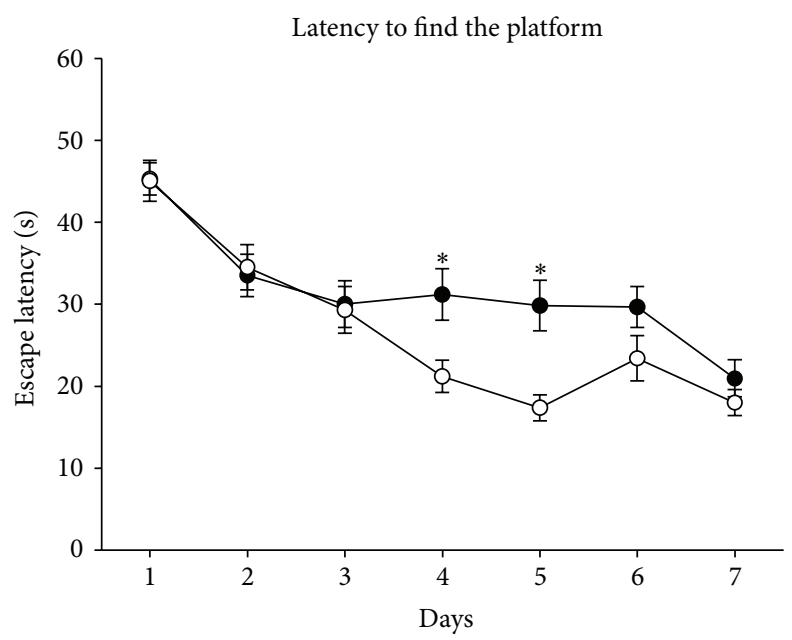

- SC

$\mathrm{OEE}$

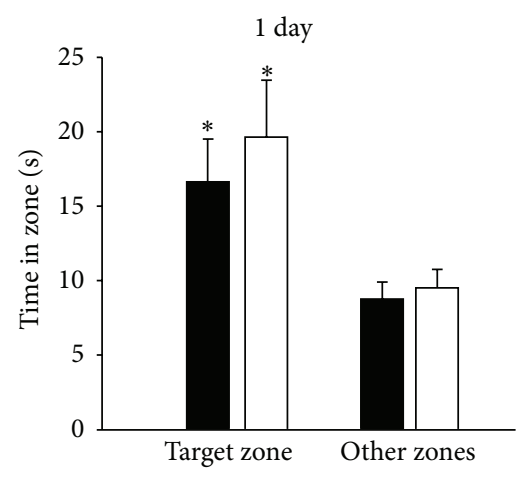

(a)
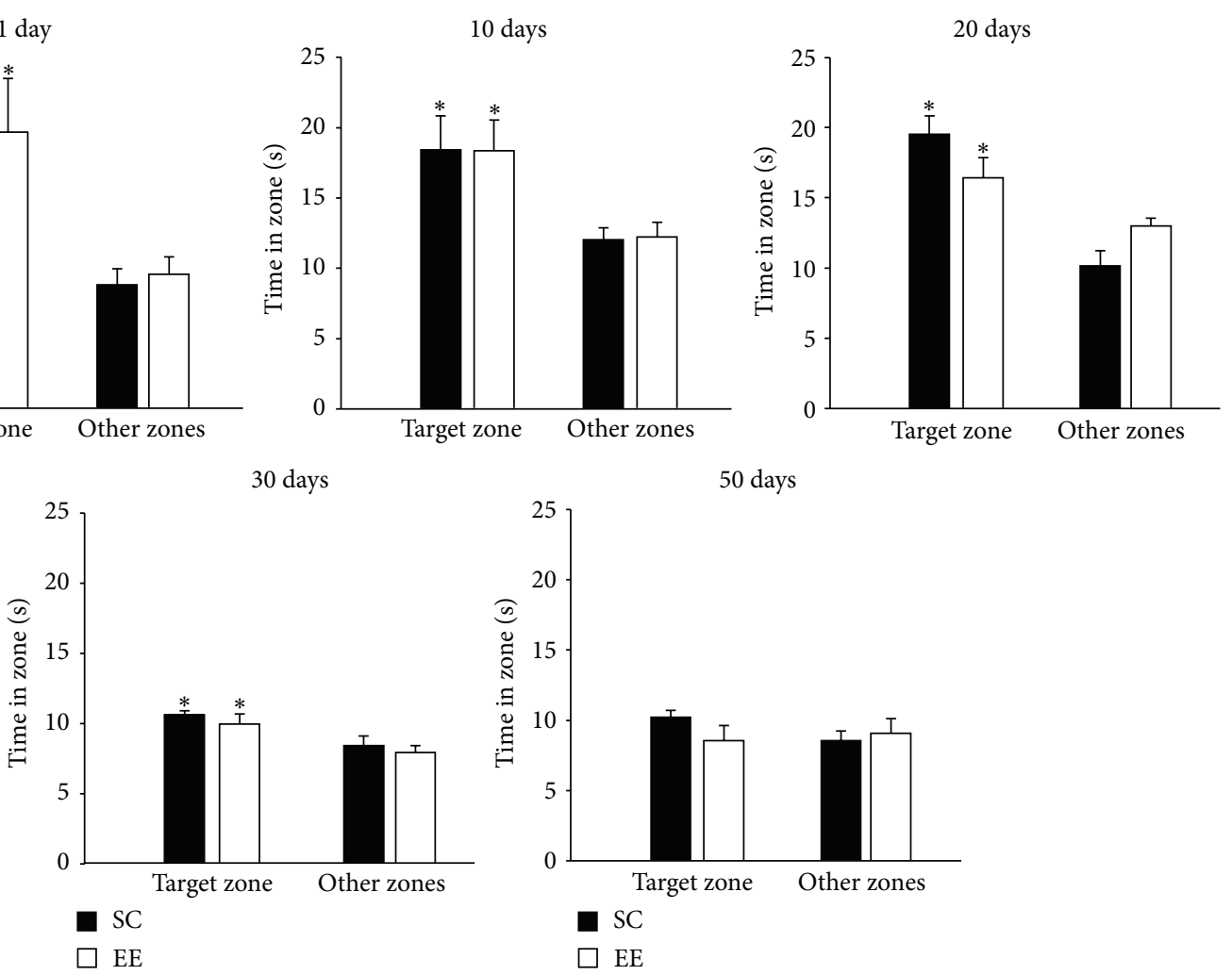

(b)

FIGURE 1: (a) Performances of SC $(n=24)$ and EE $(n=24)$ mice in the MWM. There is a significant learning effect (two-way RM ANOVA, $P=0.016$ ), and a housing condition $\times$ day interaction (two-way RM ANOVA post hoc analysis Holm-Sidak method, for day $4, P=0.005$; for day $5, P=0.001) .{ }^{*}=$ statistical significance; error bars $=$ s.e.m. (b) Evaluation of spatial memory for SC $(1$ day $n=5 ; 10$ days $n=5 ; 20$ days $n=5 ; 30 n=5,50 n=4)$ and $\operatorname{EE}(1$ day $n=5 ; 10$ days $n=5 ; 20$ days $n=5 ; 30 n=5 ; 50 n=4)$ mice. Time spent in the target zone $(T)$, where the platform was placed, versus other equivalent zones $(O)$, for recall probe tests. One-way ANOVA, post hoc analysis Holm-Sidak method, 1 day probe test: in SC group, $T$ versus $O, P=0.044$; in EE group, $T$ versus $O, P=0.036 ; 10$ days probe test: in $S C$ group, $T$ versus $O, P=0.037$; in EE group, $T$ versus $O, P=0.043 ; 20$ days probe test: in $S C$ group, $T$ versus $O, P<0.01$; in EE group, $T$ versus $O, P=0.048$; 30 days probe test: in SC group, $T$ versus $O, P=0.025$; in EE group, $T$ versus $O, P=0.048 ; 50$ days probe test: in SC group, $T$ versus $O, P=0.070$; in EE group, $T$ versus $O, P=0.226{ }^{*}=$ statistical significance; error bars $=$ s.e.m. 


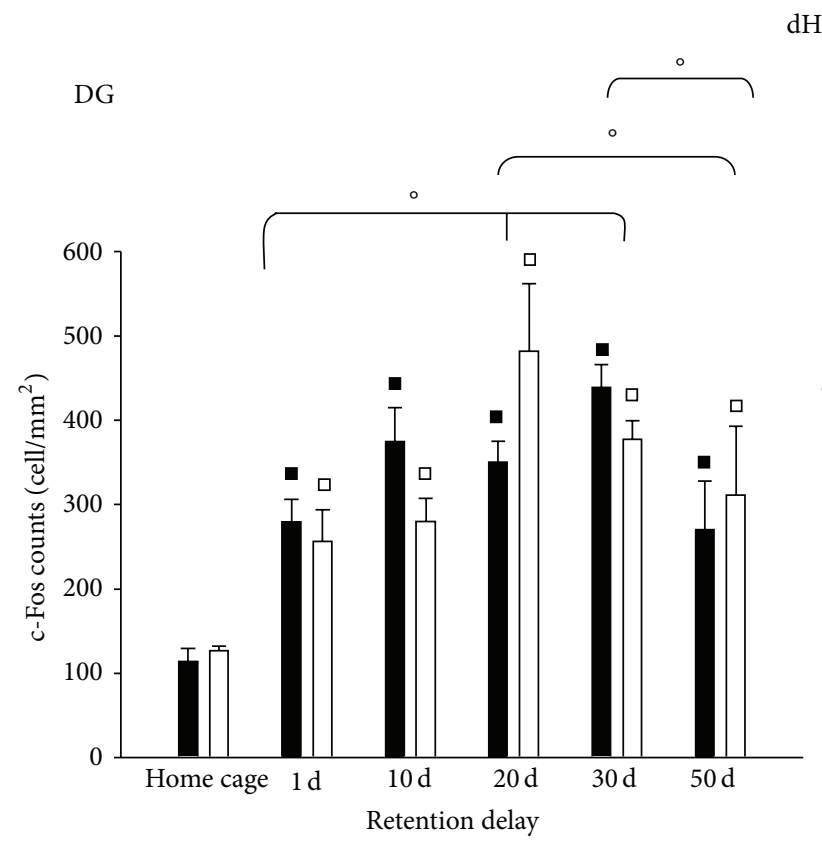

dHCP

\section{dCA1}

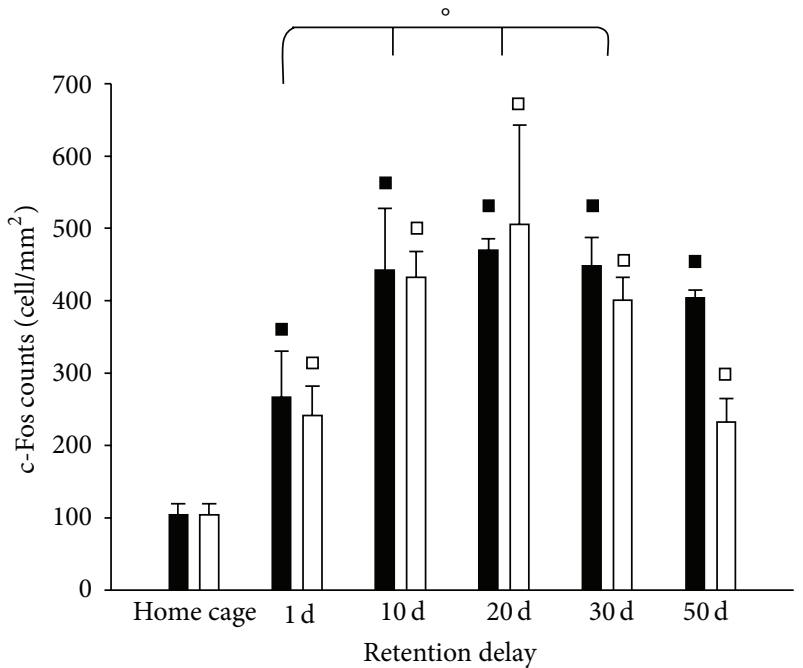

(a)

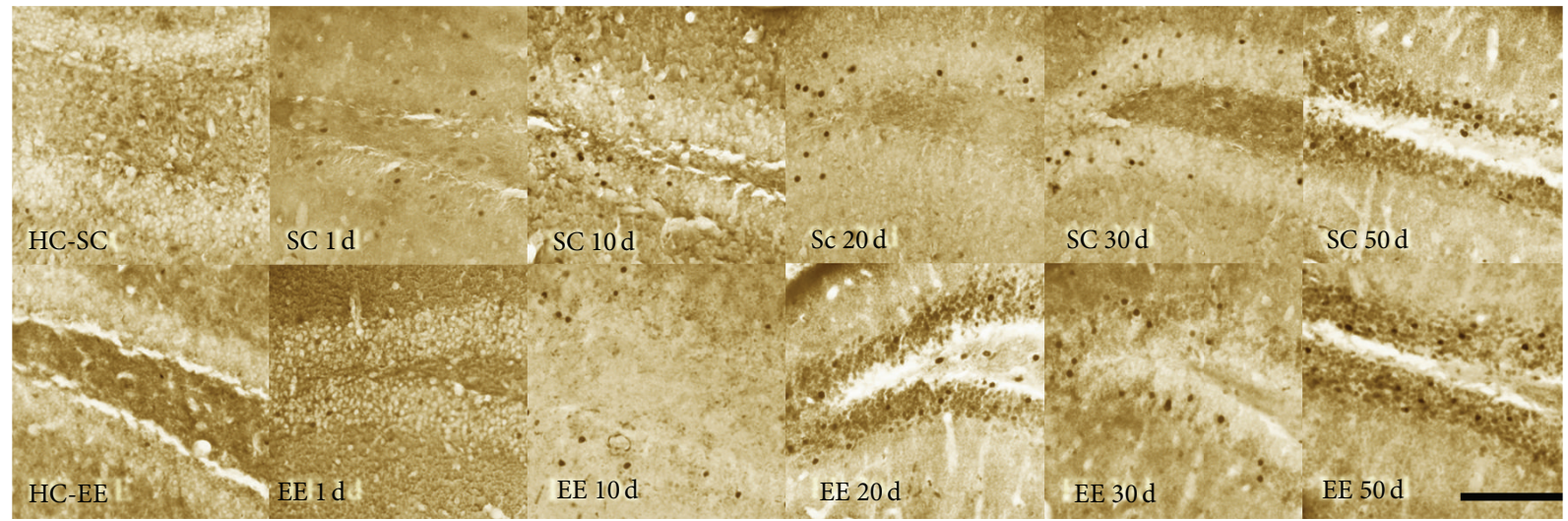

(b)

FIgURE 2: (a) c-Fos expression in subregions of dHPC for EE (open columns) and SC (filled columns) mice subjected to recall probe tests at $1,10,20,30$, and 50 days. $D G$ : two-way ANOVA post hoc analysis Holm-Sidak method, SC versus EE, $P=0.968$; HC-SC versus HC-EE, $P=0.503$; SC versus HC-SC and EE versus HC-EE $P<0.05$ for all retention intervals. Statistical differences for factor day were found between 1 and 20 days, 1 and 30 days, and 50 days versus 20 and 30 days, all $P<0.05$. $d C A 1$ : two-way ANOVA post hoc analysis Holm-Sidak method, SC versus EE, $P=0.242$; HC-SC versus HC-EE $P=0.979$. Statistical difference factor day were found between 1 and 10,20 and 30 days, all $P<0.05$. "Statistical significance between EE and HC-EE; ${ }^{\square}$ statistical significance between SC and HC-SC; ${ }^{\circ}$ statistical significance for factor day; error bars = s.e.m. (b) Representative panel of c-Fos protein expression in DG for EE and SC animals, for all recall probe tests; scale bar $100 \mu \mathrm{m}$.

investigated c-Fos expression in MEnt and in pPta, and we found that activation at 1 day in both areas was similar in EE mice, SC mice, and did not differ from that in their home-cage controls (two-way ANOVA, post hoc analysis Holm-Sidak method, all $P$ values $>0.05$ ); however, in the other probe tests performed, significant differences between EE and SC mice and between EE and HC-EE mice were found (see Figure 4 for details). Then, we observed c-Fos expression in V1 and M2, for they are connected to the dorsal stream and there is a direct monosynaptic connection between motor and visual cortices [65]. We found that EE group was statistically different from SC and HC-EE groups in M2, only in probe test performed at 20,30, and 50 days; instead, in V1, we did not find any difference between EE and SC group, only an increase in c-Fos expression for the late retention delays in both groups (see Figure 5 for details). Finally, we investigated c-Fos expression in Aul, a sensory cortex not supposed to be involved in spatial learning, and we demonstrated that activation in this area was similar in all groups (see Figure 6 for details). 

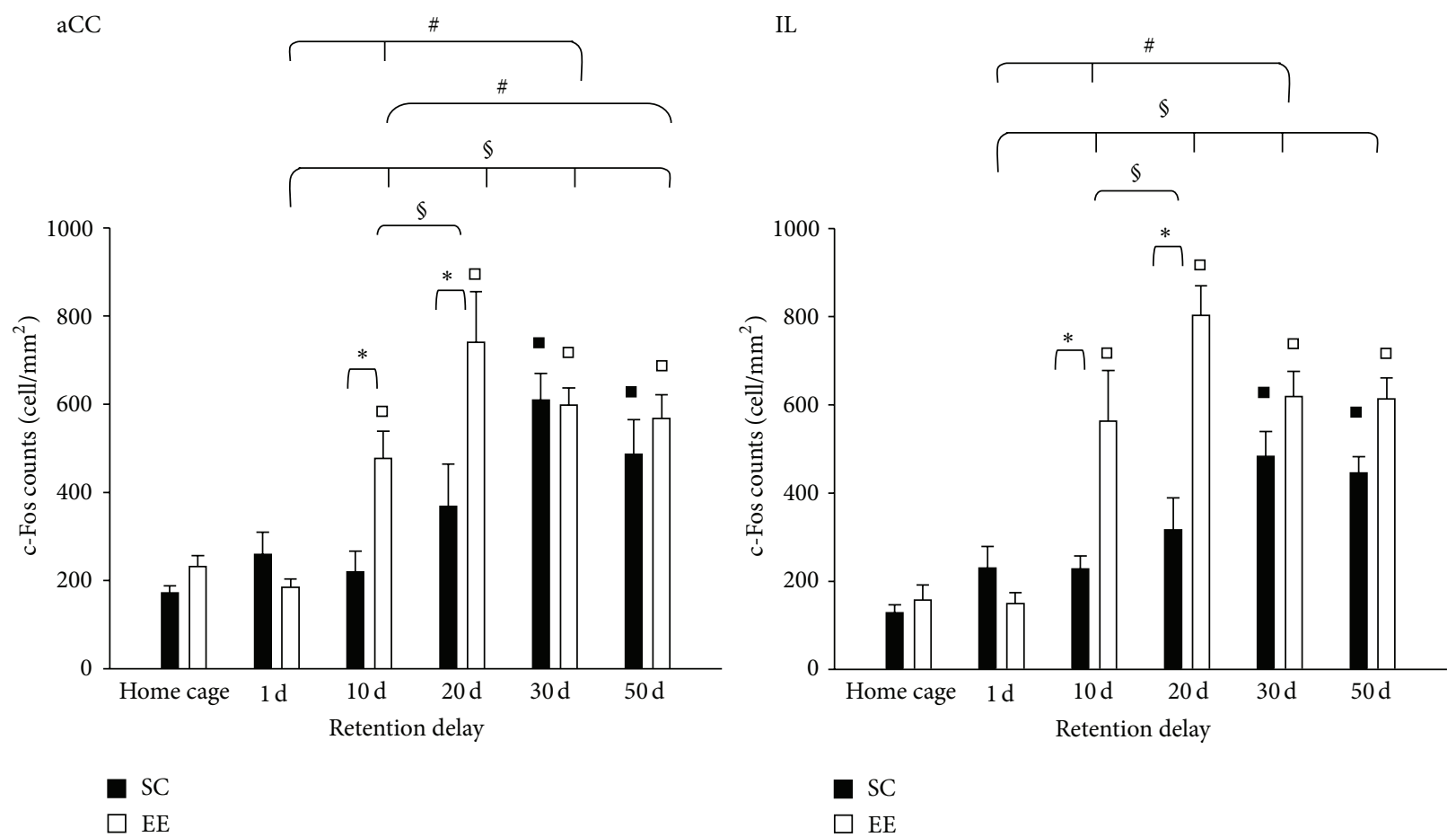

(a)



(b)

Figure 3: (a) c-Fos protein expression in aCC and IL. aCC: two-way ANOVA post hoc analysis Holm-Sidak method, HC-SC versus HC-EE $P=0.384$; statistical significance for EE versus HC-EE at 10, 20, 30, and 50 days, and for SC versus HC-SC at 30 and 50 days, all $P$ values $<$ 0.05 . Statistical difference between $\mathrm{EE}$ and $\mathrm{SC}$ at 1 day, $P=0.376$; at 10 and 20 days, $P<0.01$; at 30 days, $P=0.899$; at 50 days, $P=0.383$. Statistical difference within EE group were found between 10 and 20 days, and 1 day versus 10, 20, 30, and 50 days, all $P<0.05$. Statistical difference within SC group were found between 1 and 30 days, and 10 day versus 30 and 50 days, all $P<0.05$. IL: two-way ANOVA post hoc analysis Holm-Sidak method, HC-SC versus HC-EE, $P=0.451$; statistical significance for EE versus HC-EE at 10, 20, 30, and 50 days, and for SC versus HC-SC at 30 and 50 days, all $P$ values $<0.05$. Statistical differences between EE and SC at 1 day, $P=0.300$; at 10 and 20 days, $P<0.01$; at 30 days, $P=0.084$; at 50 days, $P=0.055$. Statistical differences within EE group were found between 10 and 20 days, and 1 day versus 10, 20,30, and 50 days, all $P<0.05$. Statistical difference within SC group were found between 1 and 30 days, and 10 day and 30 days, all $P<0.05$. "Statistical significance between EE and HC-EE; ${ }^{\square}$ statistical significance between SC and HC-SC; ${ }^{*}$ statistical significance between $\mathrm{EE}$ and SC; ${ }^{\S}=$ statistical significance for factor day within EE group; ${ }^{\#}=$ statistical significance for factor day within SC group; error bars = s.e.m. (b) Representative panel of c-Fos protein expression in aCC for EE and SC animals, for all recall probe tests; scale bar $100 \mu \mathrm{m}$.

\section{Discussion}

In this study, we provide the first evidence that EE can affect the time-dependent spatial memory system consolidation. C57BL/6 mice, housed in standard or in enriched condition, were subjected to spatial learning and then tested up to 50 days after learning to evaluate consolidation process. Using the expression of c-Fos protein as an indicator of neuronal activity in a brain-wide manner we have found indications for a difference both in the time course and in the network of 

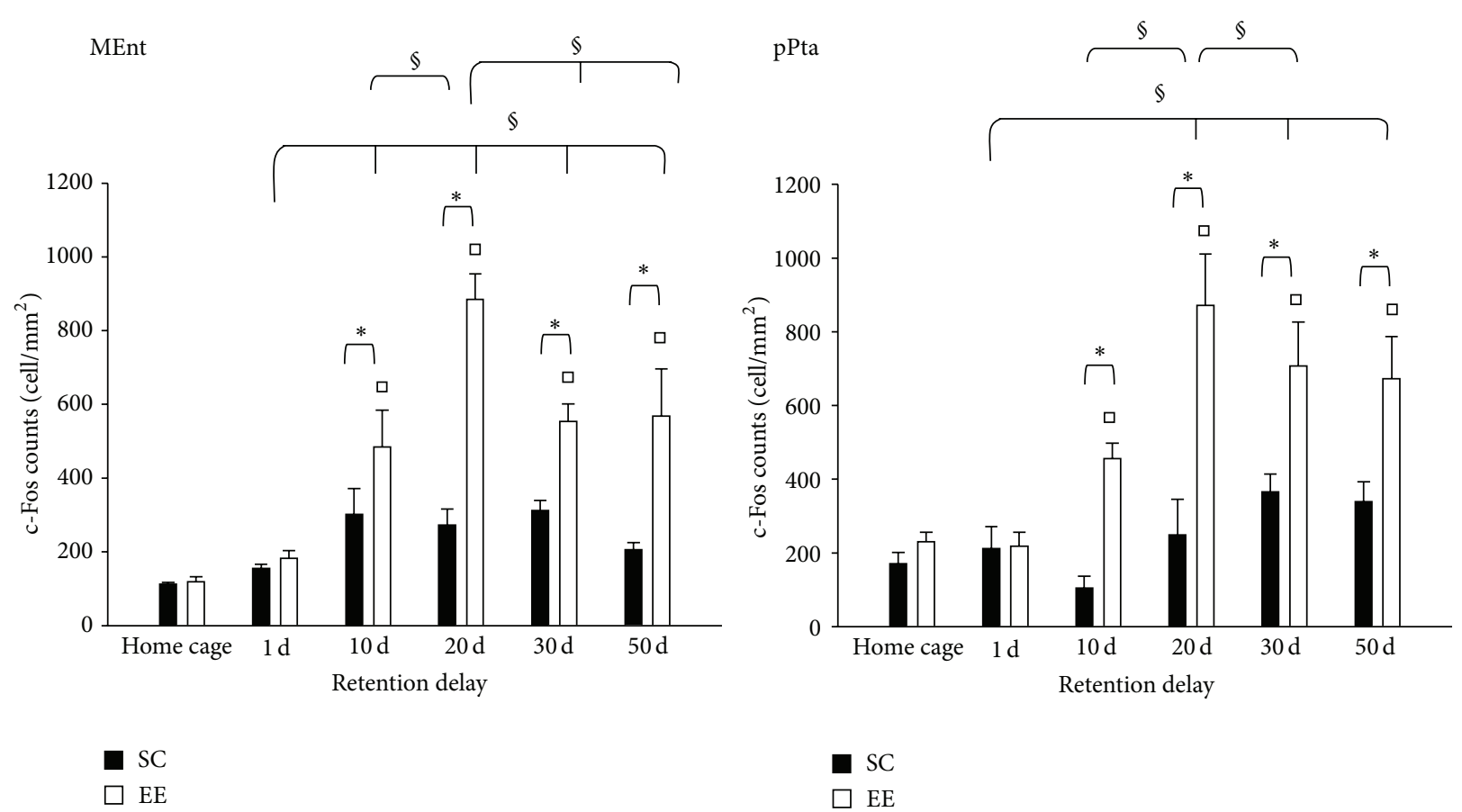

FIGURE 4: c-Fos protein expression in MEnt and pPta. MEnt: two-way ANOVA post hoc analysis Holm-Sidak method, HC-SC versus HC-EE $P=0.914$; statistical significance for EE versus HC-EE at 10, 20, 30 and 50 days. Statistical difference between EE and SC at 1 day, $P=0.782$; at 10,20,30 and 50 days, $P<0.01$. Statistical difference within EE group were found between 1 day versus 10, 20, 30 and 50 days, and 20 days versus 10, 30 and 50 days, all $P$ values $<0.05$. pPta: two-way ANOVA post hoc analysis Holm-Sidak method, HC-SC versus HC-EE $P=0.497$; statistical significance for EE versus HC-EE at 10, 20, 30 and 50 days, all $P$ values $<0.05$. Statistical difference between EE and SC at 1 day, $P=0.300$; at 10 and 20 days, $P<0.01$; at 30 days, $P=0.084$; at 50 days, $P=0.055$. Statistical difference within EE group were found between 1 day versus 20, 30 and 50 days, and 20 day versus 10 and 30 days, all $P$ values $<0.05$. Statistical difference within SC group were found between 1 and 30 days, and 10 day and 30 days, all $P$ values $<0.05$. "Statistical significance between EE and HC-EE; ${ }^{\square}$ statistical significance between SC and HC-SC; ${ }^{*}$ statistical significance between EE and SC; ${ }^{\S}=$ statistical significance for factor day within EE group; error bars = s.e.m.

cortical areas recruited for recent and remote recall between $\mathrm{EE}$ and non-EE animals.

In agreement with previous studies $[47,55]$ there was a progressive increase in c-Fos protein expression in both aCC and IL, as consolidation process proceeded. We showed that EE induces an earlier recruitment of aCC and IL with respect to SC mice; these areas were recruited following recall of spatial memory in the EE group as early as 10 days after learning, while they were recruited only 30 days after learning in SC mice. The final storage site in the cortex could be the aCC, while the IL could correlate with motivational aspects of performance, encoding other significant aspects of the environment, such as salient landmarks or preferred locations [66]. The aCC was found to be activated after remote memory recall in a number of tasks $[47,48,54,55]$, and, conversely, inactivation of the aCC disrupted recall of remote five-arm discrimination [47], contextual fear [48], and MWM [55] memories. The aCC is highly interconnected to other prefrontal regions and is reciprocally connected to sensory, motor, and limbic cortices [67, 68]; therefore, this connectivity places the aCC in favorable position, raising the possibility that this region coordinates retrieval of remote memories stored in distributed cortical networks. The earlier recruitment of aCC and IL in EE animals could imply an earlier independence of spatial memory recall from hippocampal activation in EE animals. Indeed, in animals provided with running wheels, a component of EE, block of hippocampal activation ceased to block recall of contextual fear memory at shorter time distance from learning with respect to sedentary animals [69].

In addition, we demonstrated that $\mathrm{EE}$ induces the involvement of a distributed cortical network in supporting remote spatial memory which is not activated in non EE animals. We observed that MEnt and pPta were activated following memory recall at 10 days in EE group. Both areas were included in the dorsal network for spatial navigation [60]; the entorhinal cortex contains a spatial representation of environment and plays an interface role between the hippocampus and neocortex [70]; instead, the parietal cortex, specifically the multisensory posterior region, translates coordinate information from spatial maps in the entorhinal cortex and hippocampus into egocentric representations $[59,71]$. We also investigated c-Fos protein expression in $\mathrm{V} 1$ and $\mathrm{M} 2$, and we found that $\mathrm{EE}$ group showed a greater activation in M2 than SC group, for probe test performed at 20,30, and 50 days. In V1, instead, we did not find any difference between EE and SC group, only an increase in c-Fos expression for the late retention delays in both groups; a recent study [58] showed that V1 could 


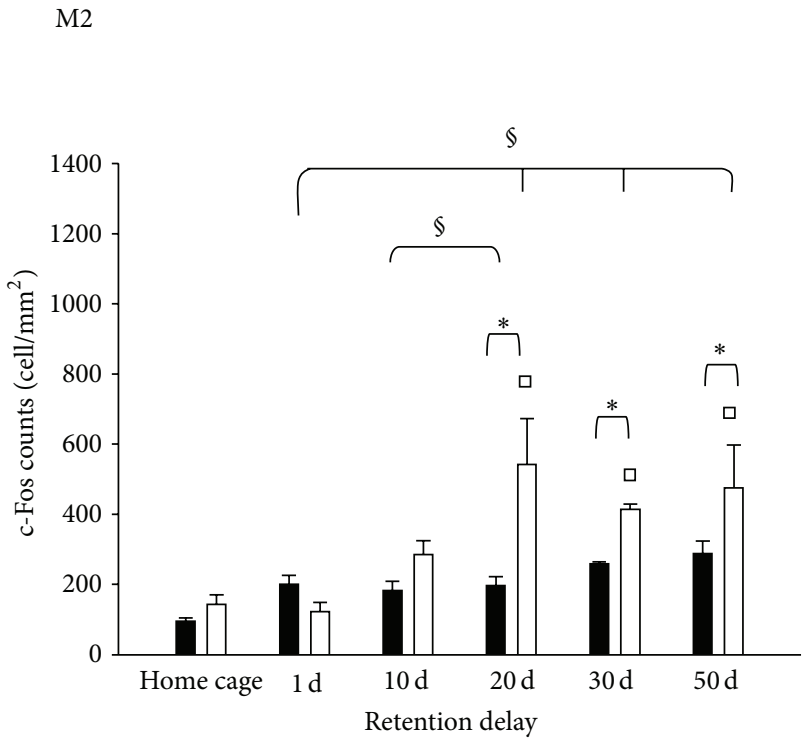

SC
V1
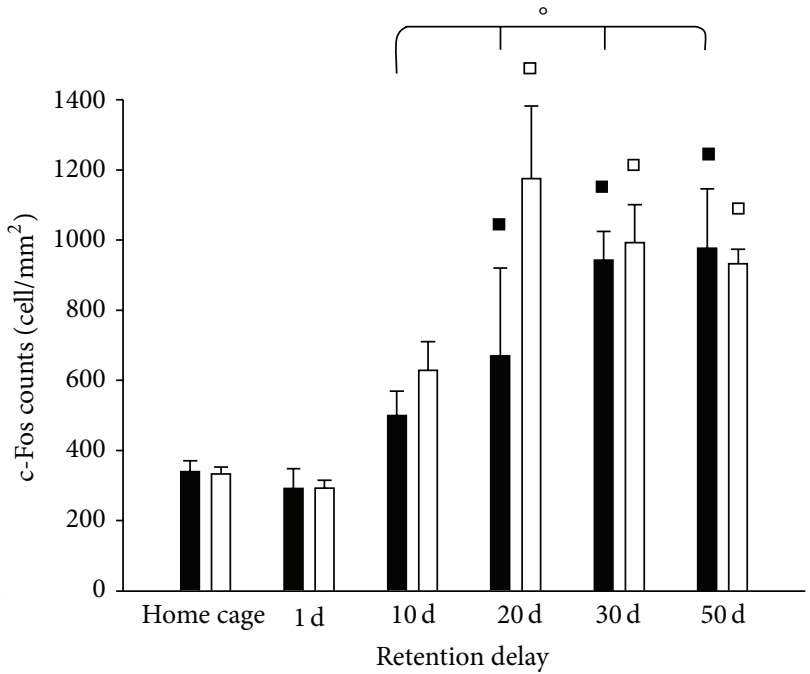

SC

$\square \mathrm{EE}$

Figure 5: c-Fos protein expression in V1 and M2. M2: two-way ANOVA post hoc analysis Holm-Sidak method, HC-SC versus HC-EE, $P=0.416$; statistical significance for EE versus HC-EE at 20,30, and 50 days. Statistical difference between EE and SC at 1 day, $P=0.288$; at 10 days, $P=0.154$; at 20,30, and 50 days, $P<0.05$. Statistical differences within EE group were found between 10 and 20 days, and 1 day versus 20, 30, and 50 days, all $P$ values < 0.05. V1: two-way ANOVA post hoc analysis Holm-Sidak method, HC-SC versus HC-EE $P=0.907$; statistical significance for EE versus HC-EE at 20, 30, and 50 days, and for SC versus HC-SC at 20, 30, and 50 days, all $P$ values $<0.05$. Statistical significance for factor day was found between 1 day versus 20,30, and 50 days, and 10 days versus 20,30 , and 50 days, all $P$ values $<0.05$. 'Statistical significance between EE and HC-EE; ${ }^{\square}$ statistical significance between SC and HC-SC; ${ }^{*}=$ statistical significance between EE and SC; ${ }^{\circ}$ statistical significance for factor day ${ }^{\S}=$ statistical significance within EE group; error bars = s.e.m.

belong to the network of fear contextual memory, although its activation did not change between recent and remote memories. In our study, the V1 pattern activation could be induced by its engagement in attentional process on account of the spatial complex task. The involvement of M2 and V1 is not surprising since they are connected to the dorsal stream [62], and a direct monosynaptic connection between motor and visual cortices was identified [65].

For the hippocampus we found no difference between EE and SC animals. Our results are consistent with the idea that hippocampus is responsible for encoding spatial memory [49-53]; its activation in remote spatial memory recall is not in agreement with studies that showed a progressive reduction in hippocampus activation with increasing retention interval $[47,48,54]$, though it is in line with the hypothesis that remote memory never becomes totally independent from the hippocampus [72]. In a more recent study, indeed, Lopez et al. [73] demonstrated that hippocampus recruitment in the recall of remote memories was influenced by the environmental conditions, such as cue saliency and complexity of the task in which memories are initially formed and subsequently recalled; thus the rich spatial details and the complexity of the training in MWM could account for the hippocampal activation found also for remote memory

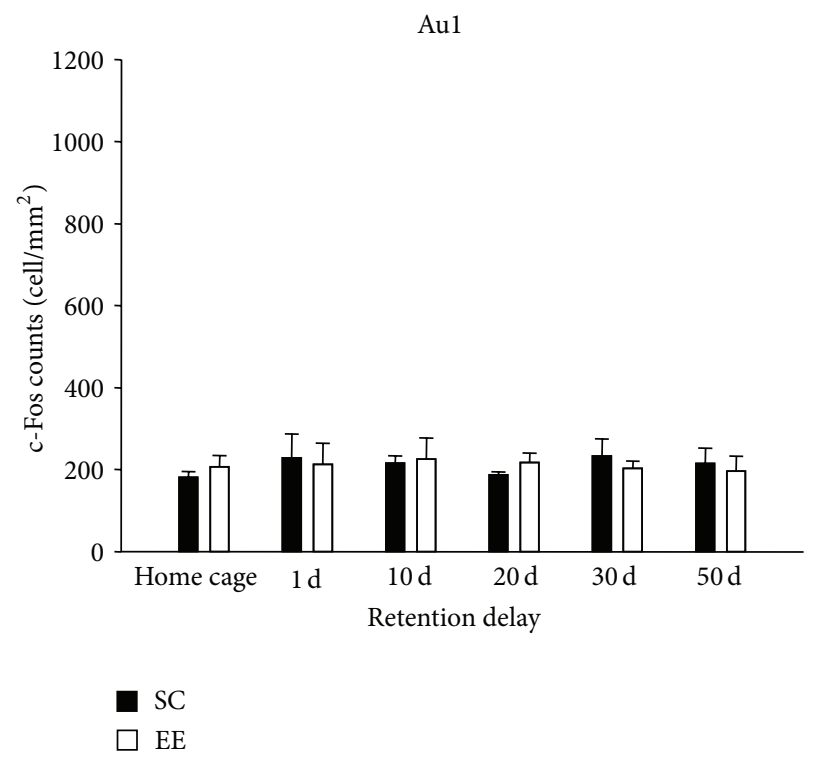

FIgURE 6: c-Fos protein expression in Aul. The data showed no difference between groups (two-way ANOVA, housing condition factor, $P=0.996$, day of probe test factor, $P=0.943$, housing condition $\times$ day of probe test interaction, $P=0.938$ ). In all probe tests, EE and SC groups did not differ from their home cage controls. 
recall. Moreover, it has been found that that precise realtime inhibition of the dorsal CA1 region, using optogenetic method, was sufficient to impair remote recall [74].

We found that EE mice were faster, considering latency parameter, in learning the position of the target platform in comparison to SC mice, but there was no significant difference between groups in the probe tests. These results are consistent with other studies in the literature which used, in rodents living in EE or provided with running wheels, intensive learning protocol for the MWM such as that used by us ( 4 daily trials for 7 consecutive days) [21]. We decided to use a behavioural protocol that maximizes the results of spatial learning task because our purpose was to examine possible differences between EE and SC groups in the time course of system consolidation without confounding effects due to differences in the results of learning, that is in the probe test, and also to be confident in the formation of a remote memory. The results of c-Fos data are an indication that the same result (a successful probe test) can be obtained through a different balance of hippocampal and cortical activation during system consolidation. Also in the Maviel and Bontempi paper [47] response accuracy in animals tested on either day 1 or 30 was similar while cortical activation strongly differed. The faster recruitment of cortical areas found in EE animals and the activation of a distributed cortical network, involving prefrontal and other associative cortices, activated in EE but not in SC animals, could suggest that the quality of the recalled memory is different in the two groups of animals; indeed, activation of prefrontal cortex has been correlated with development of the ability to create a memory that is vivid and rich in contextual details in humans [75] and activation of associative sensory cortices supports memory storage and retrieval of sensory stimuli that have acquired a behavioral salience with the experience [56].

How could EE act on the recruitment of cortical networks during system consolidation? One possibility is via its well-known action on hippocampal neurogenesis. It has been proposed that new neurons generated in the DG become functionally integrated into existing neural circuits [76]; in fact the spatial training when new neurons are more receptive to surrounding neuronal activity favored their subsequent recruitment upon remote memory retrieval $[77,78]$. Thus, these tagged adult-generated neurons, once mature, are recruited into hippocampal networks underlying remote spatial memory representation. Therefore tampering with the level of hippocampal neurogenesis could interfere in the hippocampus-only dependent period of memory. Indeed, it has been demonstrated that new hippocampal neurons were recruited into neuronal networks supporting retrieval of remote spatial memory and that the enhanced neurogenesis by voluntary running-wheel exercise sped up the disengaging from hippocampus [69]. Since EE was found to increase hippocampal neurogenesis and promote the survival of newly generated neurons [14], it is plausible that EE may accelerate the recruitment of extrahippocampal areas. In its turn, EE action on hippocampal neurogenesis is likely mediated by neurotrophins, such as brain-derived neurotrophic factor (BDNF), or by insulin growth factor-1 (IGF-1) $[9,10]$, which affect hippocampal neurogenesis and hippocampal and cortical plasticity $[14,79,80]$. IGF-1 plays an important role in cell growth and development, and it also upregulates neurogenesis in the adult hippocampus [8183]. In the adult brain, IGF-1 has been shown to mediate both the neuroprotective effects of physical exercise and the enhancement caused by exercise in hippocampal plasticity and in learning and memory [79]. Moreover IGF-1 mediates the increased expression of BDNF subsequent to EE and physical exercise [84-86]. BDNF has been shown to regulate adult hippocampal neurogenesis, to mediate EE effects on it, to modulate plasticity during learning by activating signaling pathways that modify local synaptic targets and have longterm effects on transcription, and to mediate the expression of hippocampal LTP, in both the early and late phases $[80,83$, $87,88]$.

Another nonexclusive possibility is that molecular mechanisms could "tag" the activated synapses in hippocampal and cortical networks at the time of memory encoding; this early tagging could guide the reactivation of proper hippocampalcortical connections associated with the specific memory. A recent study showed indeed that cortical tagging seems to be highly specific for precise memory trace and the impairment of early cortical tagging tampers with the postacquisition hippocampal-cortical dialogue, preventing the formation of remote memory [41]. Moreover, they demonstrated that early tagging triggers specific signaling cascades, leading to histone-tail acetylation in the cortex and that the histone deacetylase inhibitor improves remote memory retrieval [41]. Thus, early tagging acts on epigenetic modification that could mediate remote memory formation and retrieval, so $\mathrm{EE}$ could modulate the long-term memory formation and consolidation through chromatin remodeling, such as histone-tail acetylation, known to be increased in EE.

\section{Conflict of Interests}

The authors declare no conflict of interests.

\section{Authors' Contribution}

Joyce Bonaccorsi and Simona Cintoli equally contributed to this work.

\section{References}

[1] M. R. Rosenzweig, E. L. Bennett, M. Hebert, and H. Morimoto, "Social grouping cannot account for cerebral effects of enriched environments," Brain Research, vol. 153, no. 3, pp. 563-576, 1978.

[2] M. R. Rosenzweig, E. L. Bennett, and D. Krech, "Cerebral effects of environmental complexity and training among adult rats," Journal of Comparative and Physiological Psychology, vol. 57, no. 3, pp. 438-439, 1964.

[3] C. Beaulieu and M. Colonnier, "Effect of the richness of the environment on the cat visual cortex," Journal of Comparative Neurology, vol. 266, no. 4, pp. 478-494, 1987.

[4] R. L. Holloway Jr., "Dendritic branching: some preliminary results of training and complexity in rat visual cortex," Brain Research, vol. 2, no. 4, pp. 393-396, 1966. 
[5] Y. Kozorovitskiy, C. G. Gross, C. Kopil et al., "Experience induces structural and biochemical changes in the adult primate brain," Proceedings of the National Academy of Sciences of the United States of America, vol. 102, no. 48, pp. 17478-17482, 2005.

[6] A. H. Mohammed, S. W. Zhu, S. Darmopil et al., "Environmental enrichment and the brain," Progress in Brain Research, vol. 138, pp. 109-133, 2002.

[7] D. Young, P. A. Lawlor, P. Leone, M. Dragunow, and M. J. During, "Environmental enrichment inhibits spontaneous apoptosis, prevents seizures and is neuroprotective," Nature Medicine, vol. 5, no. 4, pp. 448-453, 1999.

[8] G. Kempermann, H. G. Kuhn, and F. H. Gage, "More hippocampal neurons in adult mice living in an enriched environment," Nature, vol. 386, no. 6624, pp. 493-495, 1997.

[9] B. R. Ickes, T. M. Pham, L. A. Sanders, D. S. Albeck, A. H. Mohammed, and A.-C. Granholm, "Long-term environmental enrichment leads to regional increases in neurotrophin levels in rat brain," Experimental Neurology, vol. 164, no. 1, pp. 45-52, 2000.

[10] T. M. Pham, S. Söderström, B. Winblad, and A. H. Mohammed, "Effects of environmental enrichment on cognitive function and hippocampal NGF in the non-handled rats," Behavioural Brain Research, vol. 103, no. 1, pp. 63-70, 1999.

[11] M. R. Rosenzweig, E. L. Bennett, and M. C. Diamond, "Effects of differential environments on brain anatomy and brain chemistry," Proceedings of the annual meeting of the American Psychopathological Association, vol. 56, pp. 45-56, 1967.

[12] S. Rasmuson, T. Olsson, B. G. Henriksson et al., "Environmental enrichment selectively increases 5-HT1A receptor mRNA expression and binding in the rat hippocampus," Molecular Brain Research, vol. 53, no. 1-2, pp. 285-290, 1998.

[13] F. Naka, T. Shiga, M. Yaguchi, and N. Okado, "An enriched environment increases noradrenaline concentration in the mouse brain," Brain Research, vol. 924, no. 1, pp. 124-126, 2002.

[14] H. Van Praag, G. Kempermann, and F. H. Gage, "Neural consequences of environmental enrichment," Nature Reviews Neuroscience, vol. 1, no. 3, pp. 191-198, 2000.

[15] C. Rampon, C. H. Jiang, H. Dong et al., "Effects of environmental enrichment on gene expression in the brain," Proceedings of the National Academy of Sciences of the United States of America, vol. 97, no. 23, pp. 12880-12884, 2000.

[16] A. Sale, E. Putignano, L. Cancedda et al., "Enriched environment and acceleration of visual system development," Neuropharmacology, vol. 47, no. 5, pp. 649-660, 2004.

[17] S. Landi, M. C. Cenni, L. Maffei, and N. Berardi, "Environmental enrichment effects on development of retinal ganglion cell dendritic stratification require retinal BDNF," PLoS One, vol. 2, no. 4, Article ID e346, 2007.

[18] A. Sale, M. C. Cenni, F. Clussi, E. Putignano, S. Chierzi, and L. Maffei, "Maternal enrichment during pregnancy accelerates retinal development of the fetus," PLoS One, vol. 2, no. 11, Article ID el160, 2007.

[19] A. Sale, J. F. M. Vetencourt, P. Medini et al., "Environmental enrichment in adulthood promotes amblyopia recovery through a reduction of intracortical inhibition," Nature Neuroscience, vol. 10, no. 6, pp. 679-681, 2007.

[20] L. Baroncelli, J. Bonaccorsi, M. Milanese et al., "Enriched experience and recovery from amblyopia in adult rats: impact of motor, social and sensory components," Neuropharmacology, vol. 62, no. 7, pp. 2387-2396, 2012.
[21] H. Van Praag, G. Kempermann, and F. H. Gage, "Running increases cell proliferation and neurogenesis in the adult mouse dentate gyrus," Nature Neuroscience, vol. 2, no. 3, pp. 266-270, 1999.

[22] J. C. Bennett, P. A. McRae, L. J. Levy, and K. M. Frick, "Longterm continuous, but not daily, environmental enrichment reduces spatial memory decline in aged male mice," Neurobiology of Learning and Memory, vol. 85, no. 2, pp. 139-152, 2006.

[23] S. N. Duffy, K. J. Craddock, T. Abel, and P. V. Nguyen, "Environmental enrichment modifies the PKA-dependence of hippocampal LTP and improves hippocampus-dependent memory," Learning and Memory, vol. 8, no. 1, pp. 26-34, 2001.

[24] B. A. Doty, "The effects of cage environment upon avoidance responding of aged rats," Journals of Gerontology, vol. 27, no. 3, pp. 358-360, 1972.

[25] R. A. Cummins, R. N. Walsh, and O. E. B. Olsen, "Environmentally induced changes in the brains of elderly rats," Nature, vol. 243, no. 5409, pp. 516-518, 1973.

[26] J. M. Warren, C. Zerweck, and A. Anthony, "Effects of environmental enrichment on old mice," Developmental Psychobiology, vol. 15, no. 1, pp. 13-18, 1982.

[27] G. Winocur, "Environmental influences on cognitive decline in aged rats," Neurobiology of Aging, vol. 19, no. 6, pp. 589-597, 1998.

[28] M. Soffié, K. Hahn, E. Terao, and F. Eclancher, "Behavioural and glial changes in old rats following environmental enrichment," Behavioural Brain Research, vol. 101, no. 1, pp. 37-49, 1999.

[29] G. Kempermann, D. Gast, and F. H. Gage, "Neuroplasticity in old age: sustained fivefold induction of hippocampal neurogenesis by long-term environmental enrichment," Annals of Neurology, vol. 52, no. 2, pp. 135-143, 2002.

[30] S. Kobayashi, Y. Ohashi, and S. Ando, "Effects of enriched environments with different durations and starting times on learning capacity during aging in rats assessed by a refined procedure of the Hebb-Williams maze task," Journal of Neuroscience Research, vol. 70, no. 3, pp. 340-346, 2002.

[31] K. M. Frick and S. M. Fernandez, "Enrichment enhances spatial memory and increases synaptophysin levels in aged female mice," Neurobiology of Aging, vol. 24, no. 4, pp. 615-626, 2003.

[32] P. Leal-Galicia, M. Castañeda-Bueno, R. Quiroz-Baez, and C. Arias, "Long-term exposure to environmental enrichment since youth prevents recognition memory decline and increases synaptic plasticity markers in aging," Neurobiology of Learning and Memory, vol. 90, no. 3, pp. 511-518, 2008.

[33] N. Berardi, C. Braschi, S. Capsoni, A. Cattaneo, and L. Maffei, "Environmental enrichment delays the onset of memory deficits and reduces neuropathological hallmarks in a mouse model of Alzheimer-like neurodegeneration," Journal of Alzheimer's Disease, vol. 11, no. 3, pp. 359-370, 2007.

[34] L. Baroncelli, C. Braschi, M. Spolidoro, T. Begenisic, A. Sale, and L. Maffei, "Nurturing brain plasticity: impact of environmental enrichment," Cell Death and Differentiation, vol. 17, no. 7, pp. 1092-1103, 2010.

[35] J. Nithianantharajah and A. J. Hannan, "Enriched environments, experience-dependent plasticity and disorders of the nervous system," Nature Reviews Neuroscience, vol. 7, no. 9, pp. 697-709, 2006.

[36] L. R. Squire and P. Alvarez, "Retrograde amnesia and memory consolidation: a neurobiological perspective," Current Opinion in Neurobiology, vol. 5, no. 2, pp. 169-177, 1995.

[37] J. L. McClelland, B. L. McNaughton, and R. C. O'Reilly, "Why there are complementary learning systems in the hippocampus 
and neocortex: insights from the successes and failures of connectionist models of learning and memory," Psychological Review, vol. 102, no. 3, pp. 419-457, 1995.

[38] P. J. Bayley and L. R. Squire, "The neuroscience of remote memory," Current Opinion in Neurobiology, vol. 17, no. 2, pp. 185-196, 2007.

[39] S.-H. Wang and R. G. M. Morris, "Hippocampal-neocortical interactions in memory formation, consolidation, and reconsolidation," Annual Review of Psychology, vol. 61, pp. 49-79, 2010.

[40] B. Bontempi and P. W. Frankland, "The organization of recent and remote memories," Nature Reviews Neuroscience, vol. 6, no. 2, pp. 119-130, 2005.

[41] E. Lesburguères, O. L. Gobbo, S. Alaux-Cantin, A. Hambucken, P. Trifilieff, and B. Bontempi, "Early tagging of cortical networks is required for the formation of enduring associative memory," Science, vol. 331, no. 6019, pp. 924-928, 2011.

[42] E. Shimizu, Y.-P. Tang, C. Rampon, and J. Z. Tsien, "NMDA receptor-dependent synaptic reinforcement as a crucial process for memory consolidation," Science, vol. 290, no. 5494, pp. 11701174, 2000.

[43] P. W. Frankland, C. O’Brien, M. Ohno, A. Kirkwood, and A. J. Silva, " $\alpha$-CaMKII-dependent plasticity in the cortex is required for permanent memory," Nature, vol. 411, no. 6835, pp. 309-313, 2001.

[44] M. L. Hayashi, S.-Y. Choi, B. S. Rao et al., "Altered cortical synaptic morphology and impaired memory consolidation in forebrain- specific dominant-negative PAK transgenic mice," Neuron, vol. 42, no. 5, pp. 773-787, 2004.

[45] L. Restivo, G. Vetere, B. Bontempi, and M. Ammassari-Teule, "The formation of recent and remote memory is associated with time-dependent formation of dendritic spines in the hippocampus and anterior cingulate cortex," Journal of Neuroscience, vol. 29, no. 25, pp. 8206-8214, 2009.

[46] G. Vetere, L. Restivo, C. J. Cole et al., "Spine growth in the anterior cingulate cortex is necessary for the consolidation of contextual fear memory," Proceedings of the National Academy of Sciences of the United States of America, vol. 108, no. 20, pp. 8456-8460, 2011.

[47] T. Maviel, T. P. Durkin, F. Menzaghi, and B. Bontempi, "Sites of neocortical reorganization critical for remote spatial memory," Science, vol. 305, no. 5680, pp. 96-99, 2004.

[48] P. W. Frankland, B. Bontempi, L. E. Talton, L. Kaczmarek, and A. J. Silva, "The involvement of the anterior cingulate cortex in remote contextual fear memory," Science, vol. 304, no. 5672, pp. 881-883, 2004.

[49] J. M. O'Keefe and L. Nadel, The Hippocampus as a Cognitive Map, Oxford Unversity Press, Oxford, UK, 1978.

[50] N. Burgess, E. A. Maguire, and J. O'Keefe, "The human hippocampus and spatial and episodic memory," Neuron, vol. 35, no. 4, pp. 625-641, 2002.

[51] R. G. M. Morris, E. I. Moser, G. Riedel et al., "Elements of a neurobiological theory of the hippocampus: the role of activity-dependent synaptic plasticity in memory," Philosophical Transactions of the Royal Society B, vol. 358, no. 1432, pp. 773786, 2003.

[52] K. Nakazawa, T. J. McHugh, M. A. Wilson, and S. Tonegawa, "NMDA receptors, place cells and hippocampal spatial memory," Nature Reviews Neuroscience, vol. 5, no. 5, pp. 361-372, 2004.
[53] S. Leutgeb, J. K. Leutgeb, M.-B. Moser, and E. I. Moser, "Place cells, spatial maps and the population code for memory," Current Opinion in Neurobiology, vol. 15, no. 6, pp. 738-746, 2005.

[54] B. Bontempi, C. Laurent-Demir, C. Destrade, and R. Jaffard, "Time-dependent reorganization of brain circuitry underlying long-term memory storage," Nature, vol. 400, no. 6745, pp. 671675, 1999.

[55] C. M. Teixeira, S. R. Pomedli, H. R. Maei, N. Kee, and P. W. Frankland, "Involvement of the anterior cingulate cortex in the expression of remote spatial memory," Journal of Neuroscience, vol. 26, no. 29, pp. 7555-7564, 2006.

[56] T. Sacco and B. Sacchetti, "Role of secondary sensory cortices in emotional memory storage and retrieval in rats," Science, vol. 329, no. 5992, pp. 649-656, 2010.

[57] R. Romero-Granados, Á. Fontán-Lozano, J. M. Delgado-García, and Á. M. Carrión, "From learning to forgetting: behavioral, circuitry, and molecular properties define the different functional states of the recognition memory trace," Hippocampus, vol. 20, no. 5, pp. 584-595, 2010.

[58] A. L. Wheeler, C. M. Teixeira, A. H. Wang et al., "Identification of a functional connectome for long-term fear memory in mice," PLoS Computational Biology, vol. 9, no. 1, Article ID e1002853, 2013.

[59] R. G. M. Morris, P. Garrud, J. N. P. Rawlins, and J. O’Keefe, "Place navigation impaired in rats with hippocampal lesions," Nature, vol. 297, no. 5868, pp. 681-683, 1982.

[60] E. Moser, M.-B. Moser, and P. Andersen, "Spatial learning impairment parallels the magnitude of dorsal hippocampal lesions, but is hardly present following ventral lesions," Journal of Neuroscience, vol. 13, no. 9, pp. 3916-3925, 1993.

[61] G. Paxinos and K. Franklin, The Mouse Brain in Stereotaxic Coordinates, Academic Press, San Diego, Calif, USA, 1997.

[62] Q. Wang, E. Gao, and A. Burkhalter, "Gateways of ventral and dorsal streams in mouse visual cortex," Journal of Neuroscience, vol. 31, no. 5, pp. 1905-1918, 2011.

[63] T. Hafting, M. Fyhn, S. Molden, M.-B. Moser, and E. I. Moser, "Microstructure of a spatial map in the entorhinal cortex," Nature, vol. 436, no. 7052, pp. 801-806, 2005.

[64] J. R. Whitlock, R. J. Sutherland, M. P. Witter, M.-B. Moser, and E. I. Moser, "Navigating from hippocampus to parietal cortex," Proceedings of the National Academy of Sciences of the United States of America, vol. 105, no. 39, pp. 14755-14762, 2008.

[65] A. Di Garbo, M. Mainardi, S. Chillemi, L. Maffei, and M. Caleo, "Environmental enrichment modulates cortico-cortical interactions in the mouse," PLoS One, vol. 6, no. 9, Article ID e25285, 2011.

[66] V. Hok, E. Save, P. P. Lenck-Santini, and B. Poucet, "Coding for spatial goals in the prelimbic/infralimbic area of the rat frontal cortex," Proceedings of the National Academy of Sciences of the United States of America, vol. 102, no. 12, pp. 4602-4607, 2005.

[67] H. B. M. Uylings, H. J. Groenewegen, and B. Kolb, "Do rats have a prefrontal cortex?" Behavioural Brain Research, vol. 146, no. 12, pp. 3-17, 2003.

[68] B. F. Jones, H. J. Groenewegen, and M. P. Witter, "Intrinsic connections of the cingulate cortex in the rat suggest the existence of multiple functionally segregated networks," Neuroscience, vol. 133, no. 1, pp. 193-207, 2005.

[69] T. Kitamura, Y. Saitoh, N. Takashima et al., "Adult neurogenesis modulates the hippocampus-dependent period of associative fear memory," Cell, vol. 139, no. 4, pp. 814-827, 2009. 
[70] E. I. Moser and M.-B. Moser, "A metric for space," Hippocampus, vol. 18, no. 12, pp. 1142-1156, 2008.

[71] M. Moscovitch, R. S. Rosenbaum, A. Gilboa et al., "Functional neuroanatomy of remote episodic, semantic and spatial memory: a unified account based on multiple trace theory," Journal of Anatomy, vol. 207, no. 1, pp. 35-66, 2005.

[72] L. Nadel and M. Moscovitch, "Memory consolidation, retrograde amnesia and the hippocampal complex," Current Opinion in Neurobiology, vol. 7, no. 2, pp. 217-227, 1997.

[73] J. Lopez, K. Herbeaux, B. Cosquer et al., "Context-dependent modulation of hippocampal and cortical recruitment during remote spatial memory retrieval," Hippocampus, vol. 22, no. 4, pp. 827-841, 2012.

[74] I. Goshen, M. Brodsky, R. Prakash et al., "Dynamics of retrieval strategies for remote memories," Cell, vol. 147 , no. 3, pp. 678689, 2011.

[75] N. Ofen, Y.-C. Kao, P. Sokol-Hessner, H. Kim, S. WhitfieldGabrieli, and J. D. E. Gabrieli, "Development of the declarative memory system in the human brain," Nature Neuroscience, vol. 10, no. 9, pp. 1198-1205, 2007.

[76] W. Deng, J. B. Aimone, and F. H. Gage, "New neurons and new memories: how does adult hippocampal neurogenesis affect learning and memory?" Nature Reviews Neuroscience, vol. 11, no. 5, pp. 339-350, 2010.

[77] S. Trouche, B. Bontempi, P. Roullet, and C. Rampon, "Recruitment of adult-generated neurons into functional hippocampal networks contributes to updating and strengthening of spatial memory," Proceedings of the National Academy of Sciences of the United States of America, vol. 106, no. 14, pp. 5919-5924, 2009.

[78] M. Arruda-Carvalho, M. Sakaguchi, K. G. Akers, S. A. Josselyn, and P. W. Frankland, "Posttraining ablation of adult-generated neurons degrades previously acquired memories," The Journal of Neuroscience, vol. 31, no. 42, pp. 15113-15127, 2011.

[79] N. D. Åberg, K. G. Brywe, and J. Isgaard, "Aspects of growth hormone and insulin-like growth factor-I related to neuroprotection, regeneration, and functional plasticity in the adult brain," TheScientificWorldJournal, vol. 6, pp. 53-80, 2006.

[80] E. G. Waterhouse and B. Xu, "New insights into the role of brainderived neurotrophic factor in synaptic plasticity," Molecular and Cellular Neuroscience, vol. 42, no. 2, pp. 81-89, 2009.

[81] A. J. D'Ercole, P. Ye, A. S. Calikoglu, and G. Gutierrez-Ospina, "The role of the insulin-like growth factors in the central nervous system," Molecular Neurobiology, vol. 13, no. 3, pp. 227255, 1996.

[82] J. A. D’ercole, P. Ye, and J. R. O’Kusky, “Mutant mouse models of insulin-like growth factor actions in the central nervous system," Neuropeptides, vol. 36, no. 2-3, pp. 209-220, 2002.

[83] E. Lee and H. Son, "Adult hippocampal neurogenesis and related neurotrophic factors," BMB Reports, vol. 42, no. 5, pp. 239-244, 2009.

[84] E. Carro, A. Nuñez, S. Busiguina, and I. Torres-Aleman, "Circulating insulin-like growth factor I mediates effects of exercise on the brain," Journal of Neuroscience, vol. 20, no. 8, pp. 2926-2933, 2000.

[85] Q. Ding, S. Vaynman, M. Akhavan, Z. Ying, and F. GomezPinilla, "Insulin-like growth factor I interfaces with brainderived neurotrophic factor-mediated synaptic plasticity to modulate aspects of exercise-induced cognitive function," $\mathrm{Neu}$ roscience, vol. 140, no. 3, pp. 823-833, 2006.

[86] S. Landi, F. Ciucci, L. Maffei, N. Berardi, and M. C. Cenni, "Setting the pace for retinal development: environmental enrichment acts through insulin-like growth factor 1 and brainderived neurotrophic factor," Journal of Neuroscience, vol. 29, no. 35, pp. 10809-10819, 2009.

[87] C. Rossi, A. Angelucci, L. Costantin et al., "Brain-derived neurotrophic factor (BDNF) is required for the enhancement of hippocampal neurogenesis following environmental enrichment," The European Journal of Neuroscience, vol. 24, no. 7, pp. 1850-1856, 2006.

[88] K. K. Cowansage, J. E. Ledoux, and M.-H. Monfils, "Brainderived neurotrophic factor: a dynamic gatekeeper of neural plasticity," Current Molecular Pharmacology, vol. 3, no. 1, pp. 12$29,2010$. 

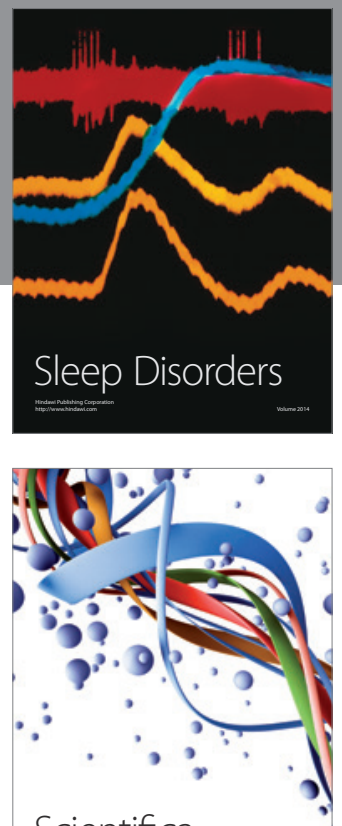

Scientifica
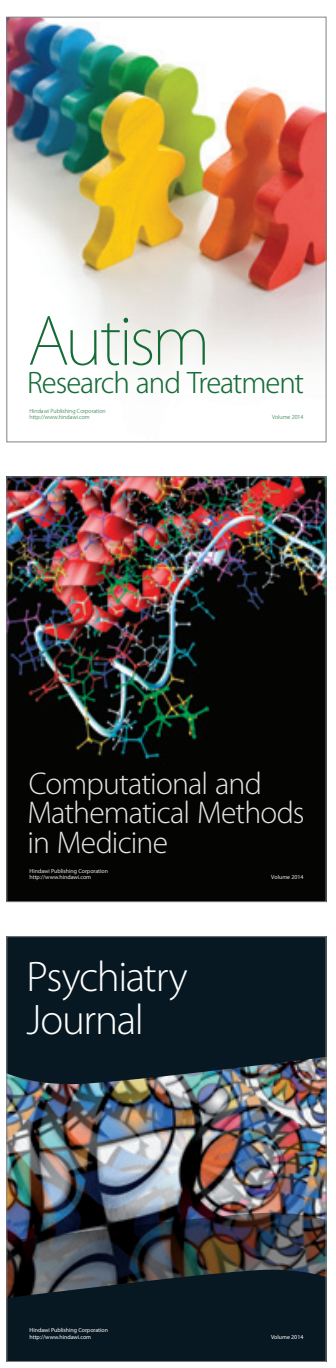
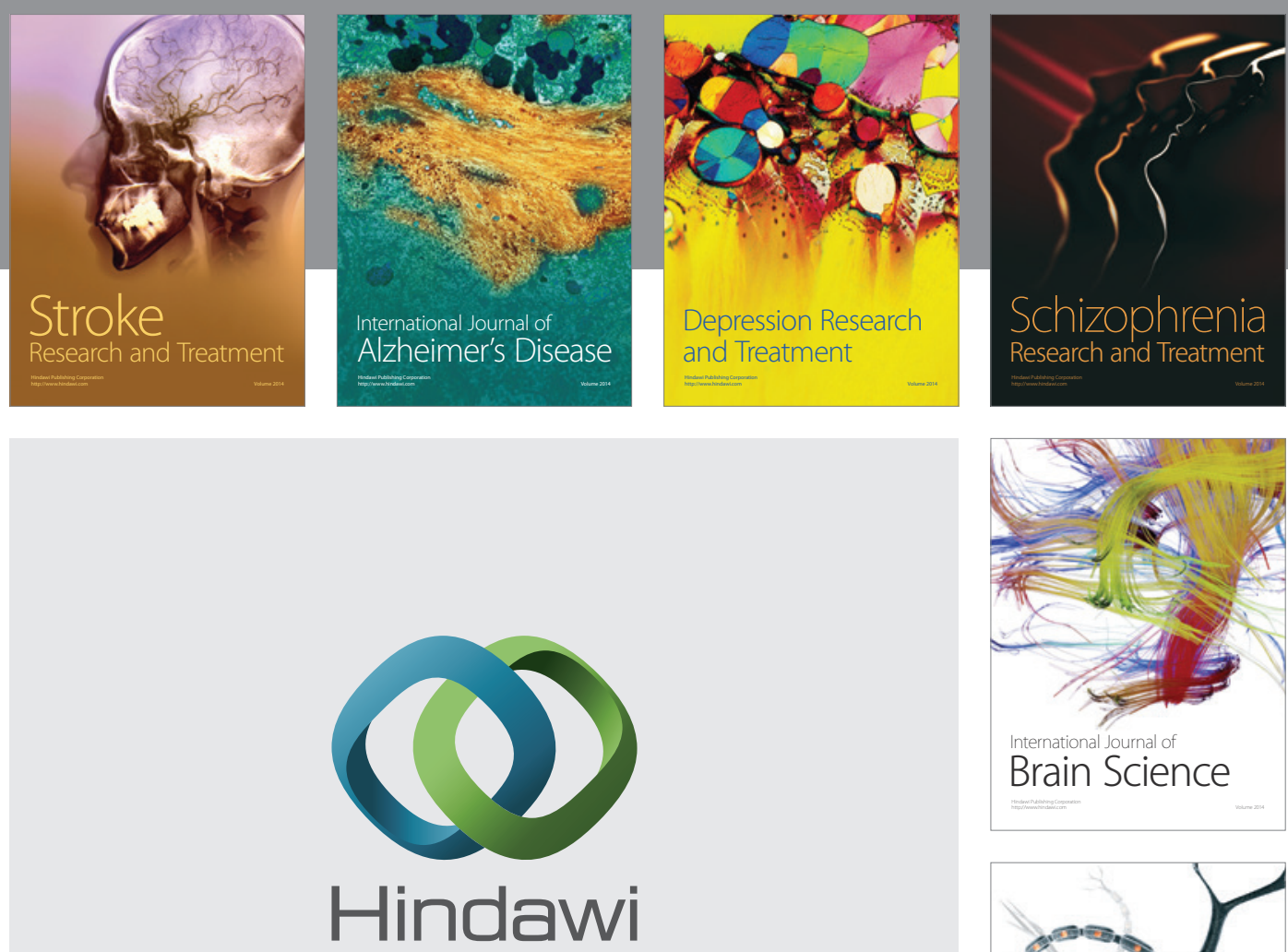

Submit your manuscripts at

http://www.hindawi.com
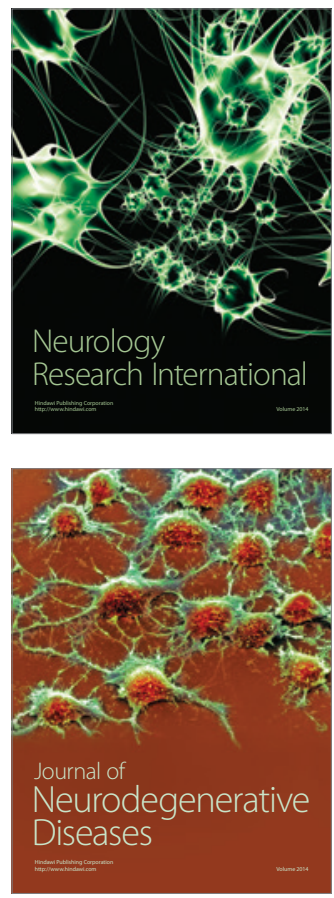

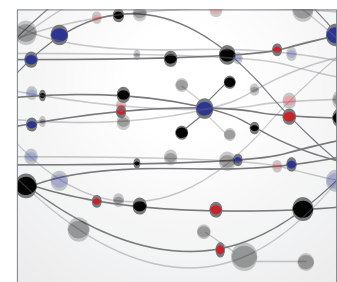

The Scientific World Journal
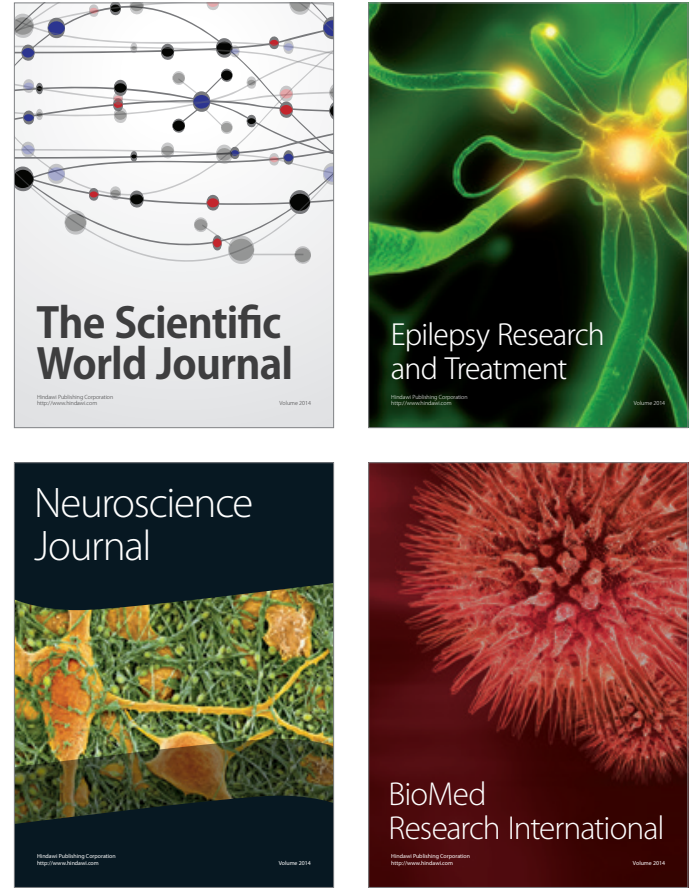

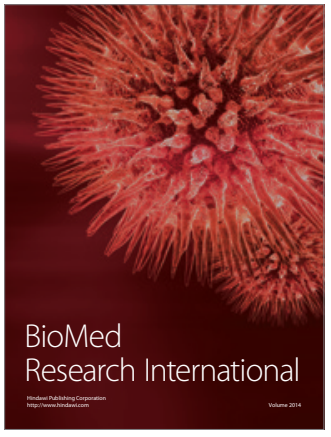

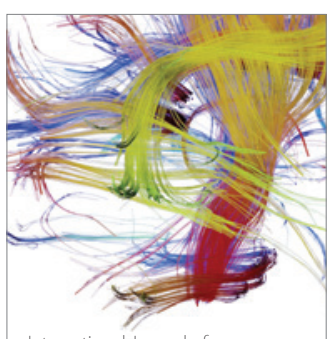

Brain Science

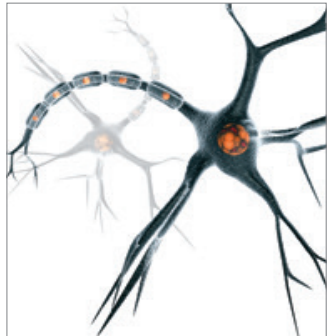

Neural Plasticity
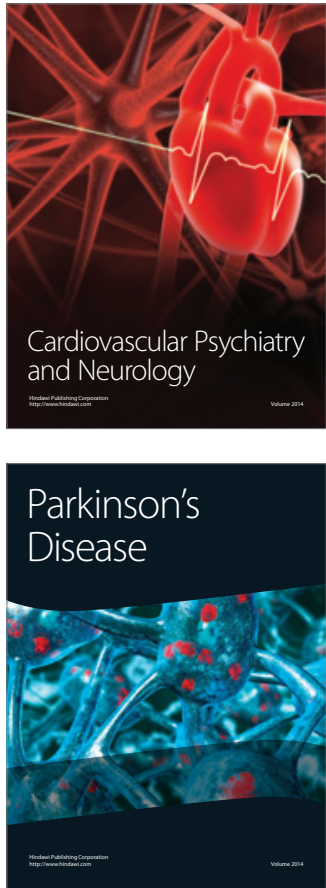